\title{
Validation of the CancerMath prognostic tool for breast cancer in Southeast Asia
}

\author{
Hui Miao ${ }^{*}$, Mikael Hartman ${ }^{1,2,3}$, Helena M. Verkooijen ${ }^{4}$, Nur Aishah Taib ${ }^{5}$, Hoong-Seam Wong ${ }^{6}$, \\ Shridevi Subramaniam ${ }^{6}$, Cheng-Har Yip ${ }^{5}$, Ern-Yu Tan ${ }^{7}$, Patrick Chan ${ }^{7}$, Soo-Chin Lee ${ }^{8}$ and Nirmala Bhoo-Pathy ${ }^{6,9,10}$
}

\begin{abstract}
Background: CancerMath is a set of web-based prognostic tools which predict nodal status and survival up to 15 years after diagnosis of breast cancer. This study validated its performance in a Southeast Asian setting.

Methods: Using Singapore Malaysia Hospital-Based Breast Cancer Registry, clinical information was retrieved from 7064 stage I to III breast cancer patients who were diagnosed between 1990 and 2011 and underwent surgery. Predicted and observed probabilities of positive nodes and survival were compared for each subgroup. Calibration was assessed by plotting observed value against predicted value for each decile of the predicted value. Discrimination was evaluated by area under a receiver operating characteristic curve (AUC) with $95 \%$ confidence interval (Cl).

Results: The median predicted probability of positive lymph nodes is $40.6 \%$ which was lower than the observed $43.6 \%$ (95\% Cl, $42.5 \%-44.8 \%)$. The calibration plot showed underestimation for most of the groups. The AUC was $0.71(95 \% \mathrm{Cl}, 0.70-0.72)$. Cancermath predicted and observed overall survival probabilities were $87.3 \%$ vs $83.4 \%$ at 5 years after diagnosis and $75.3 \%$ vs $70.4 \%$ at 10 years after diagnosis. The difference was smaller for patients from Singapore, patients diagnosed more recently and patients with favorable tumor characteristics. Calibration plot also illustrated overprediction of survival for patients with poor prognosis. The AUC for 5-year and 10-year overall survival was 0.77 (95 \% Cl: 0.75-0.79) and 0.74 (95\% Cl: 0.71-0.76).
\end{abstract}

Conclusions: The discrimination and calibration of CancerMath were modest. The results suggest that clinical application of CancerMath should be limited to patients with better prognostic profile.

Keywords: Breast cancer, CancerMath, Prognostic model, Asia

\section{Background}

Adjuvant chemotherapy and hormone therapy improve long-term survival and reduce the risk of recurrence in early breast cancer patients [1-3]. However, the benefit varies greatly from patient to patient due to biologic heterogeneity of the disease and differences in response to treatment [4, 5]. Risk of adverse effects and high cost of adjuvant therapy also make it challenging for oncologists to choose the most appropriate treatment. Therefore, several clinical tools have been developed to predict prognosis and survival benefit from treatment, using

\footnotetext{
* Correspondence: ephmh@nus.edu.sg; hui_miao@nuhs.edu.sg

'Saw Swee Hock School of Public Health, National University of Singapore and National University Health System, Tahir Foundation Building, 12 Science Drive 2, Singapore 117549, Singapore

Full list of author information is available at the end of the article
}

clinicopathological features, genetic profiles, and novel biomarkers [6].

The Nottingham Prognostic Index was the first prognostic model introduced for breast cancer patients in 1982. It includes only tumor grade, size, and nodal status for prediction of disease-free survival $[7,8]$. The widely used Adjuvant! Online (www.adjuvantonline.com) calculates 10-year overall survival and disease-free survival of patients with non-metastatic breast cancer, based on patient's age, tumor size, grade, estrogen-receptor (ER) status, nodal status, and co-morbidities. It also quantitatively predicts the absolute gain from adjuvant therapy [9]. Although it is recommended by the National Institute for Health and Clinical Excellence and widely used by oncologists [10-13], several validation studies have suggested that Adjuvant! Online is suboptimal in women 
Table 1 Observed number of patients with positive lymph nodes and predicted probability of positive nodes

\begin{tabular}{|c|c|c|c|c|c|c|c|}
\hline & \multirow{2}{*}{$\begin{array}{l}\text { Number of } \\
\text { patients }\end{array}$} & \multirow{2}{*}{$\begin{array}{l}\text { Number of patients } \\
\text { with positive lymph } \\
\text { nodes (percentage) }\end{array}$} & \multirow{2}{*}{$\begin{array}{l}\text { Predicted probability } \\
\text { of positive nodes } \\
\text { (median) }\end{array}$} & Others & 352 & $90 \%$ & $\%$ \\
\hline & & & & Unknown & 4 & $3(75.0 \%)$ & $25.1 \%$ \\
\hline Overall & 6807 & $2970(43.6 \%)$ & $40.6 \%$ & \multicolumn{4}{|l|}{ Grade } \\
\hline Ethnicity & & & & 1 & 849 & 204 (24.0 \%) & $21.8 \%$ \\
\hline Chinese & 5029 & 2062 (41.0\%) & $39.2 \%$ & 2 & 2836 & 1278 (45.1\%) & $40.6 \%$ \\
\hline Malay & 963 & $511(53.1 \%)$ & $46.0 \%$ & 3 & 2463 & 1275 (51.8 \%) & $46.4 \%$ \\
\hline Indian & 651 & $312(47.9 \%)$ & $44.7 \%$ & Unknown & 659 & $213(32.3 \%)$ & $35.9 \%$ \\
\hline
\end{tabular}

Other $\quad 164 \quad 85(51.8 \%) \quad 39.5 \%$

Country

$\begin{array}{llll}\text { Malaysia } & 3274 & 1460(44.6 \%) & 43.0 \% \\ \text { Singapore } & 3533 & 1510(42.7 \%) & 38.5 \%\end{array}$

Period of diagnosis

1990-1994 124

$58(46.8 \%) \quad 52.0 \%$

1995-1999 547

2000-2003 1744

2004-2007 2129

$258(47.2 \%)$

$41.9 \%$

$755(43.3 \%)$

$41.4 \%$

2008-2011 2263

$964(45.3 \%)$

$41.2 \%$

$935(41.3 \%)$

$38.9 \%$

Age at diagnosis

$\begin{array}{ll}0-39 & 670 \\ 40-49 & 2039 \\ 50-59 & 2145 \\ 60-69 & 1301 \\ 70+ & 652\end{array}$

$310(46.3 \%)$

$47.1 \%$

$910(44.6 \%)$

$42.9 \%$

$934(43.5 \%)$

$41.4 \%$

$546(42.0 \%)$

$36.7 \%$

$270(41.4 \%)$

$34.3 \%$

Tumor size $(\mathrm{mm})$

$\begin{array}{llll}0-20 & 2926 & 822(28.1 \%) & 26.4 \% \\ 21-50 & 3247 & 1678(51.7 \%) & 49.3 \% \\ 51+ & 634 & 470(74.1 \%) & 79.2 \%\end{array}$

ER status

$\begin{array}{cccc}\text { Negative } & 2316 & 1037(44.8 \%) & 43.5 \% \\ \text { Positive } & 4254 & 1854(43.6 \%) & 38.5 \% \\ \text { Unknown } & 237 & 79(33.3 \%) & 44.5 \% \\ \text { PR status } & & & \\ \text { Negative } & 2656 & 1195(45.0 \%) & 42.1 \% \\ \text { Positive } & 3507 & 1511(43.1 \%) & 38.5 \% \\ \text { Unknown } & 644 & 264(41.0 \%) & 44.2 \% \\ \text { Her2 status } & & & \\ \text { Negative } & 2872 & 1197(41.7 \%) & 39.2 \% \\ \text { Equivocal } & 429 & 182(42.4 \%) & 39.2 \% \\ \text { Positive } & 1315 & 662(50.3 \%) & 45.0 \% \\ \text { Unknown } & 2191 & 929(42.4 \%) & 39.6 \% \\ \text { Histology } & & & \\ \text { Ductal } & 5945 & 2681(45.1 \%) & 41.5 \% \\ \text { Lobular } & 287 & 150(52.3 \%) & 37.9 \% \\ \text { Mucinous } & 219 & 34(15.5 \%) & 10.7 \%\end{array}$

Table 1 Observed number of patients with positive lymph nodes and predicted probability of positive nodes (Continued)

younger than 40 years and older than 75 years $[14,15]$. The model was recently validated in Malaysia, Korea, and Taiwan, where it was shown to substantially overestimate actual survival [16-18]. CancerMath (http:// www.lifemath.net/cancer/) is the latest web-based prognostic tool, which takes human epidermal growth factor receptor 2 (HER2) status into account [19]. It was established based on the binary biological model of cancer metastasis and the parameters were derived from the Surveillance, Epidemiology and End-Result (SEER) registry in the United States [20]. CancerMath provides information on overall survival, conditional survival (the likelihood of surviving given being alive after a certain number of years) and benefit of systemic treatment for each of the first 15 years after diagnosis. This model also estimates probability of positive lymph nodes and nipple involvement. Validation study has shown comparable results between CancerMath and Adjuvant! Online [19]. However this new tool has not been validated outside the United States. Given the differences in underlying distribution of prognostic factors and life expectancy between Asia and the United States [21-23], direct application without any correction may not generate reliable prediction. The aim of the study is to validate this model in the Singapore Malaysia Hospital-Based Breast Cancer Registry, demonstrating its predictive performance for different subgroups and determining its calibration and discrimination.

\section{Methods}

Women diagnosed with pathological stage I to III breast cancer according to American Joint Committee on Cancer Staging Manual sixth edition, who underwent surgery, were identified from the Singapore Malaysia Hospital-Based Breast Cancer Registry, which combined databases from three public tertiary hospitals. The breast cancer registry at National University Hospital (NUH) in Singapore collects information on breast cancer patients diagnosed since 1990. The Tan Tock Seng Hospital (TTSH) registry registers patients diagnosed from 2001 onwards. The University Malaya Medical Centre (UMMC), located in Kuala Lumpur, Malaysia, has 
Table 2 Observed and predicted 5-year overall survival from outcome calculator, stratified by patients' characteristics

\begin{tabular}{|c|c|c|c|c|c|c|c|}
\hline & $N$ & $\begin{array}{l}\text { Observed deaths } \\
\text { in } 5 \text { years }\end{array}$ & $\begin{array}{l}\text { Predicted deaths } \\
\text { in } 5 \text { years }\end{array}$ & $\begin{array}{l}\text { Mortality Ratio } \\
(95 \% \text { Cl) }\end{array}$ & $\begin{array}{l}\text { Observed 5-year } \\
\text { survival (\%) (std err) }\end{array}$ & $\begin{array}{l}\text { Predicted 5-year } \\
\text { survival (median) (\%) }\end{array}$ & $\begin{array}{l}\text { Absolute difference } \\
(\%)(95 \% \mathrm{Cl})\end{array}$ \\
\hline Overall & 4517 & 752 & 667 & $1.13(1.05,1.21)$ & $83.4(0.006)$ & 87.3 & $3.9(2.7,5.1)$ \\
\hline \multicolumn{8}{|l|}{ Ethnicity } \\
\hline Chinese & 3340 & 488 & 478 & $1.02(0.93,1.12)$ & $85.4(0.006)$ & 88.0 & $2.6(1.4,3.8)$ \\
\hline Malay & 654 & 143 & 104 & $1.38(1.16,1.62)$ & $78.1(0.016)$ & 85.8 & $7.7(4.6,10.8)$ \\
\hline Indian & 430 & 109 & 71 & $1.54(1.26,1.85)$ & $74.7(0.021)$ & 85.1 & $10.4(6.3,14.5)$ \\
\hline Other & 93 & 12 & 14 & $0.86(0.44,1.50)$ & $87.1(0.035)$ & 87.3 & $0.2(-6.7,7.1)$ \\
\hline \multicolumn{8}{|l|}{ Country } \\
\hline Malaysia & 2143 & 423 & 331 & $1.28(1.16,1.41)$ & $80.3(0.009)$ & 86.1 & $5.8(4.0,7.6)$ \\
\hline Singapore & 2374 & 329 & 336 & $0.98(0.88,1.09)$ & $86.1(0.007)$ & 88.6 & $2.5(1.1,3.9)$ \\
\hline \multicolumn{8}{|c|}{ Period of diagnosis } \\
\hline 1990-1994 & 140 & 41 & 22 & $1.86(1.34,2.53)$ & $70.7(0.038)$ & 85.9 & $15.2(7.8,22.6)$ \\
\hline 1995-1999 & 564 & 116 & 75 & $1.55(1.28,1.86)$ & $79.8(0.017)$ & 87.9 & $8.1(4.8,11.4)$ \\
\hline $2000-2003$ & 1800 & 279 & 261 & $1.07(0.95,1.20)$ & $84.5(0.009)$ & 87.8 & $3.3(1.5,5.1)$ \\
\hline 2004-2007 & 2013 & 316 & 309 & $1.02(0.91,1.14)$ & $84.3(0.008)$ & 87.2 & $2.9(1.3,4.5)$ \\
\hline \multicolumn{8}{|c|}{ Age at diagnosis } \\
\hline $0-39$ & 493 & 101 & 64 & $1.58(1.29,1.92)$ & $79.5(0.018)$ & 88.8 & $9.3(5.8,12.8)$ \\
\hline $40-49$ & 1430 & 172 & 163 & $1.06(0.90,1.23)$ & $88.0(0.009)$ & 90.6 & $2.6(0.8,4.4)$ \\
\hline $50-59$ & 1412 & 224 & 194 & $1.15(1.01,1.32)$ & $84.1(0.010)$ & 88.2 & $4.1(2.1,6.1)$ \\
\hline $60-69$ & 776 & 126 & 130 & $0.97(0.81,1.15)$ & $83.8(0.013)$ & 85.1 & $1.3(-1.2,3.8)$ \\
\hline $70+$ & 406 & 129 & 117 & $1.10(0.92,1.31)$ & $68.2(0.023)$ & 73.9 & $5.7(1.2,10.2)$ \\
\hline \multicolumn{8}{|c|}{ Tumor size (mm) } \\
\hline $0-20$ & 1889 & 151 & 173 & $0.87(0.74,1.02)$ & $92.0(0.006)$ & 92.9 & $0.9(-0.3,2.1)$ \\
\hline $21-50$ & 2180 & 438 & 374 & $1.17(1.06,1.29)$ & $79.9(0.009)$ & 84.8 & $4.9(3.1,6.7)$ \\
\hline $51+$ & 448 & 163 & 121 & $1.35(1.15,1.57)$ & $63.6(0.023)$ & 73.6 & $10.0(5.5,14.5)$ \\
\hline \multicolumn{8}{|c|}{ Number of positive nodes } \\
\hline 0 & 2408 & 196 & 238 & $0.82(0.71,0.95)$ & $91.9(0.006)$ & 91.7 & $-0.2(-1.4,1.0)$ \\
\hline $1-3$ & 1068 & 195 & 165 & $1.18(1.02,1.36)$ & $81.7(0.012)$ & 85.9 & $4.2(1.8,6.6)$ \\
\hline $4-9$ & 533 & 159 & 122 & $1.30(1.11,1.52)$ & $70.2(0.020)$ & 78.0 & $7.8(3.9,11.7)$ \\
\hline $10+$ & 354 & 170 & 116 & $1.47(1.25,1.70)$ & $52.0(0.027)$ & 67.4 & $15.4(10.1,20.7)$ \\
\hline Unknown & 154 & 32 & 27 & $1.19(0.81,1.67)$ & $79.2(0.033)$ & 86.6 & $7.4(0.9,13.9)$ \\
\hline \multicolumn{8}{|l|}{ ER status } \\
\hline Negative & 1595 & 392 & 268 & $1.46(1.32,1.61)$ & $75.4(0.011)$ & 85.2 & $9.8(7.6,12.0)$ \\
\hline Positive & 2668 & 309 & 367 & $0.84(0.75,0.94)$ & $88.4(0.006)$ & 88.8 & $0.4(-0.8,1.6)$ \\
\hline Unknown & 254 & 51 & 33 & $1.55(1.15,2.03)$ & $79.9(0.025)$ & 88.6 & $8.7(3.8,13.6)$ \\
\hline \multicolumn{8}{|l|}{ PR status } \\
\hline Negative & 1674 & 382 & 289 & $1.32(1.19,1.46)$ & $77.2(0.010)$ & 84.8 & $7.6(5.6,9.6)$ \\
\hline Positive & 2174 & 241 & 285 & $0.85(0.74,0.96)$ & $88.9(0.007)$ & 89.5 & $0.6(-0.8,2.0)$ \\
\hline Unknown & 669 & 129 & 93 & $1.39(1.16,1.65)$ & $80.7(0.015)$ & 87.4 & $6.7(3.8,9.6)$ \\
\hline \multicolumn{8}{|l|}{ Her2 status } \\
\hline Negative & 1483 & 208 & 210 & $0.99(0.86,1.13)$ & $86.0(0.009)$ & 88.0 & $2.0(0.2,3.8)$ \\
\hline Equivocal & 118 & 19 & 19 & $1.00(0.60,1.56)$ & $83.9(0.034)$ & 87.4 & $3.5(-3.2,10.2)$ \\
\hline Positive & 790 & 172 & 147 & $1.17(1.00,1.36)$ & $78.2(0.015)$ & 83.0 & $4.8(1.9,7.7)$ \\
\hline Unknown & 2126 & 353 & 292 & $1.21(1.09,1.34)$ & $83.4(0.008)$ & 88.7 & $5.3(3.7,6.9)$ \\
\hline
\end{tabular}


Table 2 Observed and predicted 5-year overall survival from outcome calculator, stratified by patients' characteristics (Continued)

\begin{tabular}{|c|c|c|c|c|c|c|c|}
\hline \multicolumn{8}{|l|}{ Histology } \\
\hline Ductal & 3951 & 696 & 597 & $1.17(1.08,1.26)$ & $82.4(0.006)$ & 87.0 & $4.6(3.4,5.8)$ \\
\hline Lobular & 180 & 17 & 26 & $0.65(0.38,1.05)$ & $90.6(0.022)$ & 87.5 & $-3.1(-7.4,1.2)$ \\
\hline Mucinous & 156 & 10 & 14 & $0.71(0.34,1.31)$ & $93.6(0.020)$ & 94.7 & $1.1(-2.8,5.0)$ \\
\hline Others & 227 & 29 & 30 & $0.97(0.65,1.39)$ & $87.2(0.022)$ & 89.5 & $2.3(-2.0,6.6)$ \\
\hline Unknown & 3 & 0 & 0 & - & 100 & 86.8 & -13.2 \\
\hline \multicolumn{8}{|l|}{ Grade } \\
\hline 1 & 552 & 20 & 44 & $0.45(0.28,0.70)$ & $96.4(0.008)$ & 94.7 & $-1.7(-3.3,-0.1)$ \\
\hline 2 & 1882 & 261 & 265 & $0.98(0.87,1.11)$ & $86.1(0.008)$ & 88.2 & $2.1(0.5,3.7)$ \\
\hline 3 & 1591 & 402 & 288 & $1.40(1.26,1.54)$ & $74.7(0.011)$ & 84.3 & $9.6(7.4,11.8)$ \\
\hline Unknown & 492 & 69 & 70 & $0.99(0.77,1.25)$ & $86.0(0.016)$ & 87.4 & $1.4(-1.7,4.5)$ \\
\hline
\end{tabular}

Numbers marked in bold indicate statistically significant difference at the $95 \%$ confidence level

prospectively collected data on breast cancer patients diagnosed since 1993 [24]. No consent was needed and ethics approval was obtained from Domain Specific Review Board under National Healthcare Group in Singapore and Medical Ethics Committee under UMMC. The consolidated registry included information on ethnicity, age and date of diagnosis, histologically determined tumor size, number of positive lymph nodes, ER and progesterone receptor (PR) status (positive defined as $1 \%$ or more positively stained tumor cells at NUH or $10 \%$ or more positively stained tumor cells at TTSH and UMMC, negative, or unknown), HER2 status based on fluorescence in situ hybridization (FISH) or immunohistochemistry (IHC) if FISH was not performed (positive defined as FISH positive or IHC score of $3+$, negative defined as FISH negative or IHC scored of 0 or $1+$, equivocal defined as IHC score of $2+$, or unknown), histological type (ductal, lobular, mucinous, others, or unknown), grade (1, 2, 3, or unknown), type of surgery (no surgery, mastectomy, breast conserving surgery, or unknown), chemotherapy (yes, no or unknown), hormone therapy (yes, no, or unknown), and radiotherapy (yes, no, or unknown). Detailed chemotherapeutic treatment regimens were only available for UMMC patients. For chemotherapy, cyclophosphamide, methotrexate and fluorouracil (CMF) was categorized as first generation regimen and fluorouracil, epirubicin and cyclophosphamide (FEC), and doxorubicin and cyclophosphamide $(\mathrm{AC})$ followed by paclitaxel were second generation. Docetaxel, doxorubicin and cyclophosphamide (TAC), and FEC followed by docetaxel were categorized as third generation. Hormone therapy was categorized into five groups: tamoxifen, aromatase inhibitors (AI), tamoxifen followed by AI, ovarian ablation, and ovarian ablation plus tamoxifen. Vital status was obtained from the hospitals' medical records and ascertained by linkage to death registries in both countries. Patients diagnosed until $31^{\text {st }}$ December 2011 were followed up from date of diagnosis until date of death or date of last fellow-up, whichever came first.
Date of last follow-up was $1^{\text {st }}$ March 2013 for UMMC, $31^{\text {st }}$ July 2013 for NUH, and $1^{\text {st }}$ October 2012 for TTSH. Male patients, patients with unknown age at diagnosis and tumor size were excluded from this analysis as these two were essential predictors for all four CancerMath calculators.

Javascript codes of all four CancerMath calculators which contained predetermined parameters and mathematical equations were exported on $9^{\text {th }}$ Nov 2013 from its website by selecting "view-> source" in the browser menu. The script was then transcribed into $\mathrm{R}$ script to allow calculation for a group of patients. For nodal status calculator, patient's age, tumor size, ER and PR status, histological type, and grade were used by the program to calculate probability of positive nodes for each patient. Overall mortality risk at each year up to 15 year after diagnoses was predicted by outcome calculator, based on age, tumor size, number of positive nodes, grade, histological type, ER, PR, and HER2 status. Effect of hormone and chemotherapeutic regimen on overall mortality was further adjusted by the therapy calculator and number of years since diagnosis were considered in the conditional survival calculator. Results from $\mathrm{R}$ script and website were crosschecked with a random subset of 20 patients to verify the accuracy of R script. Histological type recorded as others was re-categorized as unknown. If HER2 status was equivocal based on IHC and FISH was not performed, HER2 status was treated as unknown. Evidence of recurrence was set as unknown for conditional survival calculation.

In total, 7064 female breast cancer patients were included. Only cases with known nodal status $(N=6807)$ were included for validation of nodal status calculator and their individual probability of positive lymph nodes was calculated. For outcome calculator, two separate subsets of patients with minimum 5-year follow up (UMMC and NUH patients diagnosed in 2007 and earlier and TTSH patient diagnosed in 2006 and earlier, $N=4517$ ) and patients with 10-year follow-up UMMC and NUH cases diagnosed in 2002 and earlier, $N=1649$ ) 
Table 3 Observed and predicted 10-year overall survival from outcome calculator, stratified by patients' characteristics

\begin{tabular}{|c|c|c|c|c|c|c|c|}
\hline & $N$ & $\begin{array}{l}\text { Observed death } \\
\text { in } 10 \text { years }\end{array}$ & $\begin{array}{l}\text { Predicted death } \\
\text { in } 10 \text { years }\end{array}$ & $\begin{array}{l}\text { Mortality Ratio } \\
(95 \% \mathrm{Cl})\end{array}$ & $\begin{array}{l}\text { Observed 10-year } \\
\text { survival (\%)(std err) }\end{array}$ & $\begin{array}{l}\text { Predicted 10-year } \\
\text { survival (median) (\%) }\end{array}$ & $\begin{array}{l}\text { Absolute difference } \\
(\%)(95 \% \mathrm{Cl})\end{array}$ \\
\hline Overall & 1649 & 488 & 454 & $1.07(0.98,1.17)$ & $70.4(0.011)$ & 75.3 & $4.9(2.7,7.1)$ \\
\hline \multicolumn{8}{|l|}{ Ethnicity } \\
\hline Chinese & 1201 & 318 & 318 & $1.00(0.89,1.12)$ & $73.5(0.013)$ & 76.8 & $3.3(0.8,5.8)$ \\
\hline Malay & 251 & 100 & 74 & $1.35(1.10,1.64)$ & $60.2(0.031)$ & 72.3 & $12 \cdot 1(6.0,18.2)$ \\
\hline Indian & 174 & 64 & 55 & $1.16(0.90,1.49)$ & $63.2(0.037)$ & 69.9 & $6.7(-0.6,14.0)$ \\
\hline Other & 23 & 6 & 7 & $0.86(0.31,1.87)$ & $73.9(0.092)$ & 77.1 & $3.2(-14.8,21.2)$ \\
\hline \multicolumn{8}{|l|}{ Country } \\
\hline Malaysia & 983 & 341 & 284 & $1.20(1.08,1.34)$ & $65.3(0.015)$ & 73.3 & $8.0(5.1,10.9)$ \\
\hline Singapore & 666 & 147 & 170 & $0.86(0.73,1.02)$ & $77.9(0.016)$ & 77.9 & $0.0(-3.1,3.1)$ \\
\hline \multicolumn{8}{|c|}{ Period of diagnosis } \\
\hline 1990-1994 & 140 & 56 & 42 & $1.33(1.01,1.73)$ & $60.0(0.041)$ & 72.5 & $12.5(4.5,20.5)$ \\
\hline 1995-1999 & 564 & 187 & 148 & $1.26(1.09,1.46)$ & $66.8(0.020)$ & 76.0 & $9.2(5.3,13.1)$ \\
\hline 2000-2002 & 945 & 245 & 264 & $0.93(0.82,1.05)$ & $74.1(0.014)$ & 75.9 & $1.8(-0.9,4.5)$ \\
\hline \multicolumn{8}{|c|}{ Age at diagnosis } \\
\hline $0-39$ & 232 & 82 & 58 & $1.41(1.12,1.75)$ & $64.7(0.031)$ & 77.3 & $12.6(6.5,18.7)$ \\
\hline $40-49$ & 576 & 137 & 130 & $1.05(0.88,1.25)$ & $76.2(0.018)$ & 80.2 & $4.0(0.5,7.5)$ \\
\hline $50-59$ & 493 & 141 & 129 & $1.09(0.92,1.29)$ & $71.4(0.020)$ & 76.4 & $5.0(1.1,8.9)$ \\
\hline $60-69$ & 254 & 78 & 86 & $0.91(0.72,1.13)$ & $69.3(0.029)$ & 68.4 & $-0.9(-6.6,4.8)$ \\
\hline $70+$ & 94 & 50 & 50 & $1.00(0.74,1.32)$ & $46.8(0.051)$ & 50.1 & $3.3(-6.7,13.3)$ \\
\hline \multicolumn{8}{|c|}{ Tumor size (mm) } \\
\hline $0-20$ & 653 & 118 & 109 & $1.08(0.90,1.30)$ & $81.9(0.015)$ & 86.8 & $4.9(2.0,7.8)$ \\
\hline $21-50$ & 831 & 283 & 262 & $1.08(0.96,1.21)$ & $65.9(0.016)$ & 70.6 & $4.7(1.6,7.8)$ \\
\hline $51+$ & 165 & 87 & 82 & $1.06(0.85,1.31)$ & $47.3(0.039)$ & 50.6 & $3.3(-4.3,10.9)$ \\
\hline \multicolumn{8}{|c|}{ Number of positive nodes } \\
\hline 0 & 867 & 147 & 161 & $0.91(0.77,1.07)$ & $83.0(0.013)$ & 84.0 & $1.0(-1.5,3.5)$ \\
\hline $1-3$ & 407 & 143 & 120 & $1.19(1.00,1.40)$ & $64.9(0.024)$ & 72.1 & $7.2(2.5,11.9)$ \\
\hline $4-9$ & 215 & 112 & 93 & $1.20(0.99,1.45)$ & $47.9(0.034)$ & 58.2 & $10.3(3.6,17.0)$ \\
\hline $10+$ & 104 & 71 & 62 & $1.15(0.89,1.44)$ & $31.7(0.046)$ & 39.9 & $8.2(-0.8,17.2)$ \\
\hline Unknown & 56 & 15 & 17 & $0.88(0.49,1.46)$ & $73.2(0.059)$ & 73.5 & $0.3(-11.3,11.9)$ \\
\hline \multicolumn{8}{|l|}{ ER status } \\
\hline Negative & 637 & 224 & 197 & $1.14(0.99,1.30)$ & $64.8(0.019)$ & 71.5 & $6.7(3.0,10.4)$ \\
\hline Positive & 816 & 205 & 206 & $1.00(0.86,1.14)$ & $74.9(0.015)$ & 78.2 & $3.3(0.4,6.2)$ \\
\hline Unknown & 196 & 59 & 51 & $1.16(0.88,1.49)$ & $69.9(0.033)$ & 76.8 & $6.9(0.4,13.4)$ \\
\hline \multicolumn{8}{|l|}{ PR status } \\
\hline Negative & 485 & 160 & 153 & $1.05(0.89,1.22)$ & $67.0(0.021)$ & 70.7 & $3.7(-0.4,7.8)$ \\
\hline Positive & 564 & 128 & 136 & $0.94(0.79,1.12)$ & $77.3(0.018)$ & 79.9 & $2.6(-0.9,6.1)$ \\
\hline Unknown & 600 & 200 & 165 & $1.21(1.05,1.39)$ & $66.7(0.019)$ & 74.1 & $7.4(3.7,11.1)$ \\
\hline \multicolumn{8}{|l|}{ Her2 status } \\
\hline Negative & 269 & 72 & 66 & $1.09(0.85,1.37)$ & $73.2(0.027)$ & 78.3 & $5.1(-0.2,10.4)$ \\
\hline Equivocal & 13 & 6 & 4 & $1.50(0.55,3.26)$ & $53.8(0.138)$ & 65.5 & $11.7(-15.3,38.7)$ \\
\hline Positive & 335 & 113 & 110 & $1.03(0.85,1.24)$ & $66.3(0.026)$ & 69.1 & $2.8(-2.3,7.9)$ \\
\hline Unknown & 1032 & 297 & 273 & $1.09(0.97,1.22)$ & $71.2(0.014)$ & 76.8 & $5.6(2.9,8.3)$ \\
\hline
\end{tabular}


Table 3 Observed and predicted 10-year overall survival from outcome calculator, stratified by patients' characteristics (Continued)

\begin{tabular}{|c|c|c|c|c|c|c|c|}
\hline \multicolumn{8}{|l|}{ Histology } \\
\hline Ductal & 1418 & 445 & 401 & $1.11(1.01,1.22)$ & $68.6(0.012)$ & 74.4 & $5.8(3.4,8.2)$ \\
\hline Lobular & 78 & 18 & 21 & $0.86(0.51,1.35)$ & $76.9(0.048)$ & 75.7 & $-1.2(-10.6,8.2)$ \\
\hline Mucinous & 59 & 9 & 9 & $1.00(0.46,1.90)$ & $84.7(0.047)$ & 91.2 & $6.5(-2.7,15.7)$ \\
\hline Others & 91 & 16 & 22 & $0.73(0.42,1.18)$ & $82.4(0.040)$ & 77.7 & $-4.7(-12.5,3.1)$ \\
\hline Unknown & 3 & 0 & 1 & - & 100 & 74.4 & -25.6 \\
\hline \multicolumn{8}{|l|}{ Grade } \\
\hline 1 & 200 & 22 & 31 & $0.71(0.44,1.07)$ & $89.0(0.022)$ & 89.3 & $0.3(-4.0,4.6)$ \\
\hline 2 & 668 & 188 & 176 & $1.07(0.92,1.23)$ & $71.9(0.017)$ & 77.1 & $5.2(1.9,8.5)$ \\
\hline 3 & 510 & 196 & 172 & $1.14(0.99,1.31)$ & $61.6(0.022)$ & 70.0 & $8.4(4.1,12.7)$ \\
\hline Unknown & 271 & 82 & 76 & $1.08(0.86,1.34)$ & $69.7(0.028)$ & 73.3 & $3.6(-1.9,9.1)$ \\
\hline
\end{tabular}

Numbers marked in bold indicate statistically significant difference at the $95 \%$ confidence level

were selected for comparison of observed and predicted survival. As NUH and TTSH did not collect details of hormone therapy and chemotherapy regimen data before 2006, therapy calculator was only validated for UMMC patients with minimum 5-year follow up $(N=1538)$.

\section{Statistical analysis}

\section{Nodal status calculator}

Observed and predicted probability of positive lymph nodes were compared. Calibration was assessed by dividing the data into deciles based on the predicted probability of positive nodes and then plotting the observed probability of positive nodes against means of predicted probability for each decile. A 45 degree diagonal line was plotted to illustrate perfect agreement. Discrimination of nodal status calculator was evaluated by area under the curve (AUC) in receiver operating characteristic analysis. A value of 0.5 indicates no discrimination and a value of 1.0 means perfect discrimination.

\section{Outcome and therapy calculator}

Ratio of observed and predicted numbers of death within 5 years and 10 years of diagnosis were calculated as mortality ratio (MR) with $95 \%$ confidence interval (CI) constructed by exact procedure [25]. MR was also calculated for different subgroups by country, period of diagnosis, age, race, and other clinical characteristics. Observed 5-year and 10-year survival rates were compared with the median predicted survival from CancerMath. A difference of less than $3 \%$ would be considered reliable enough for clinical use as 10-year survival benefit of $3-5 \%$ is an indication for adjuvant chemotherapy [26]. The relationship of average 5 -year and 10-year predicted survival and observed 5-year and 10-year survival was illustrated by the calibration plot. Discrimination of outcome and therapy calculator was evaluated by AUC using dataset with minimum 5-year and 10-year followup accordingly. Outcome calculator was further evaluated using concordance index (c-index) proposed by
Harrell et al. for the entire dataset regardless of followup time [27]. C-index is the probability of correctly distinguishing patient who survives longer within a random pair of patients [27]. Like for the AUC, a c-index of 0.5 indicates no discrimination and a c-index of 1.0 means perfect discrimination.

\section{Conditional survival calculator}

For patients who survived two years after diagnosis, predicted 5-year survival was compared with observed 5year survival. Similarly predicted 10-year survival was compared with observed 10-year survival for patients who survived 5 years and 7 years respectively. Discrimination was evaluated by AUC.

\section{Results}

In total, 7064 female breast cancer patients were included. Tables 1, 2, 3 and 4 present clinical characteristics of 6807 patients with nodal status, 4517 patients with minimum 5-year follow-up, 1649 patients with 10-year followup, and 1538 patients with detailed treatment data and minimum of 5-years follow-up, respectively.

\section{Nodal status calculator}

A total of 6807 patients with nodal status data were selected for validation of nodal status calculator. In this dataset, $43.6 \%$ patients $(n=2970)(95 \%$ CI, $42.5 \%-44.8 \%$ ) had at least one positive lymph node and the median predicted probability was $40.6 \%$. CancerMath underestimated the probability of positive node for most of the subgroups (Table 1). The calibration plot (Fig. 1) also illustrated underestimation except for the last two deciles. The discrimination of this calculator was fair, with AUC of 0.71 (95\% CI, 0.70-0.72).

\section{Outcome calculator}

The observed number of deaths within 5 years after diagnosis was significantly higher than the predicted 
Table 4 Observed and predicted 5-year overall survival from therapy calculator, stratified by patients' characteristics

\begin{tabular}{|c|c|c|c|c|c|c|c|}
\hline & $N$ & $\begin{array}{l}\text { Observed death } \\
\text { in } 5 \text { years }\end{array}$ & $\begin{array}{l}\text { Predicted death } \\
\text { in } 5 \text { years }\end{array}$ & $\begin{array}{l}\text { Mortality Ratio } \\
(95 \% \mathrm{Cl})\end{array}$ & $\begin{array}{l}\text { Observed 5-year } \\
\text { survival (\%)(std err) }\end{array}$ & $\begin{array}{l}\text { Predicted 5-year } \\
\text { survival (median) (\%) }\end{array}$ & $\begin{array}{l}\text { Absolute difference } \\
(\%)(95 \% \mathrm{Cl})\end{array}$ \\
\hline Overall & 1538 & 286 & 173 & $1.65(1.47,1.86)$ & $81.4(0.010)$ & 89.8 & $8.4(6.4,10.4)$ \\
\hline \multicolumn{8}{|l|}{ Ethnicity } \\
\hline Chinese & 1052 & 167 & 113 & $1.48(1.26,1.72)$ & $84.1(0.011)$ & 90.4 & $6.3(4.1,8.5)$ \\
\hline Malay & 264 & 62 & 30 & $2.07(1.58,2.65)$ & $76.5(0.026)$ & 89.4 & $12.9(7.8,18.0)$ \\
\hline Indian & 212 & 54 & 29 & $1.86(1.40,2.43)$ & $74.5(0.030)$ & 87.2 & $12.7(6.8,18.6)$ \\
\hline Other & 10 & 3 & 1 & $3.00(0.62,8.77)$ & $70.0(0.145)$ & 88.2 & $18.2(-10.2,46.6)$ \\
\hline \multicolumn{8}{|c|}{ Period of diagnosis } \\
\hline 1990-1994 & 95 & 39 & 14 & $2.79(1.98,3.81)$ & $58.9(0.05)$ & 86.8 & $27.9(18.1,37.7)$ \\
\hline 1995-1999 & 374 & 93 & 40 & $2.33(1.88,2.85)$ & $75.1(0.022)$ & 90.9 & $15.8(11.5,20.1)$ \\
\hline 2000-2003 & 568 & 91 & 63 & $1.44(1.16,1.77)$ & $84.0(0.015)$ & 89.7 & $5.7(2.8,8.6)$ \\
\hline 2004-2007 & 501 & 63 & 56 & $1.13(0.86,1.44)$ & $87.4(0.015)$ & 90.2 & $2.8(-0.1,5.7)$ \\
\hline \multicolumn{8}{|c|}{ Age at diagnosis } \\
\hline 0-39 & 205 & 55 & 17 & $3.24(2.44,4.21)$ & $73.2(0.031)$ & 92.6 & $19.4(13.3,25.5)$ \\
\hline $40-49$ & 515 & 74 & 41 & $1.80(1.42,2.27)$ & $85.6(0.015)$ & 92.9 & $7.3(4.4,10.2)$ \\
\hline $50-59$ & 449 & 86 & 50 & $1.72(1.38,2.12)$ & $80.8(0.019)$ & 89.4 & $8.6(4.9,12.3)$ \\
\hline $60-69$ & 271 & 43 & 40 & $1.08(0.78,1.45)$ & $84.1(0.022)$ & 86.1 & $2.0(-2.3,6.3)$ \\
\hline $70+$ & 98 & 28 & 24 & $1.17(0.78,1.69)$ & $71.4(0.046)$ & 77.4 & $6.0(-3.0,15.0)$ \\
\hline \multicolumn{8}{|c|}{ Tumor size (mm) } \\
\hline $0-20$ & 547 & 51 & 39 & $1.31(0.97,1.72)$ & $90.7(0.012)$ & 94.2 & $3.5(1.1,5.9)$ \\
\hline $21-50$ & 813 & 170 & 102 & $1.67(1.43,1.94)$ & $79.1(0.014)$ & 88.5 & $9.4(6.7,12.1)$ \\
\hline $51+$ & 178 & 65 & 32 & $2.03(1.57,2.59)$ & $63.5(0.036)$ & 82.8 & $19.3(12.2,26.4)$ \\
\hline \multicolumn{8}{|c|}{ Number of positive nodes } \\
\hline 0 & 806 & 72 & 70 & $1.03(0.80,1.30)$ & $91.1(0.010)$ & 92.4 & $1.3(-0.7,3.3)$ \\
\hline $1-3$ & 389 & 83 & 46 & $1.80(1.44,2.24)$ & $78.7(0.021)$ & 89.4 & $10.7(6.6,14.8)$ \\
\hline $4-9$ & 192 & 64 & 30 & $2.13(1.64,2.72)$ & $66.7(0.034)$ & 85.8 & $19.1(12.4,25.8)$ \\
\hline $10+$ & 123 & 61 & 23 & $2.65(2.03,3.41)$ & $50.4(0.045)$ & 82.3 & $31.9(23.1,40.7)$ \\
\hline Unknown & 28 & 6 & 4 & $1.50(0.55,3.26)$ & 78.6 (0.078) & 90.6 & $12.0(-3.3,27.3)$ \\
\hline \multicolumn{8}{|l|}{ ER status } \\
\hline Negative & 528 & 146 & 73 & $2.00(1.69,2.35)$ & $72.3(0.019)$ & 87.2 & $14.9(11.2,18.6)$ \\
\hline Positive & 850 & 99 & 82 & $1.21(0.98,1.47)$ & $88.4(0.011)$ & 91.7 & $3.3(1.1,5.5)$ \\
\hline Unknown & 160 & 41 & 18 & $2.28(1.63,3.09)$ & 74.4 (0.035) & 89.8 & $15.4(8.5,22.3)$ \\
\hline \multicolumn{8}{|l|}{ PR status } \\
\hline Negative & 423 & 106 & 57 & $1.86(1.52,2.25)$ & $74.9(0.021)$ & 87.4 & $12.5(8.4,16.6)$ \\
\hline Positive & 586 & 73 & 58 & $1.26(0.99,1.58)$ & $87.5(0.014)$ & 91.6 & $4.1(1.4,6.8)$ \\
\hline Unknown & 529 & 107 & 58 & $1.84(1.51,2.23)$ & $79.8(0.017)$ & 90.2 & $10.4(7.1,13.7)$ \\
\hline \multicolumn{8}{|l|}{ Her2 status } \\
\hline Negative & 665 & 78 & 68 & $1.15(0.91,1.43)$ & $88.3(0.012)$ & 91.1 & $2.8(0.4,5.2)$ \\
\hline Equivocal & 35 & 7 & 4 & $1.75(0.70,3.61)$ & $80.0(0.068)$ & 89.9 & $9.9(-3.4,23.2)$ \\
\hline Positive & 418 & 84 & 53 & $1.58(1.26,1.96)$ & $79.9(0.020)$ & 87.9 & $8.0(4.1,11.9)$ \\
\hline Unknown & 420 & 117 & 48 & $2.44(2.02,2.92)$ & $72.1(0.022)$ & 89.7 & $17.6(13.3,21.9)$ \\
\hline \multicolumn{8}{|l|}{ Histology } \\
\hline Ductal & 1346 & 270 & 155 & $1.74(1.54,1.96)$ & $79.9(0.011)$ & 89.6 & $9.7(7.5,11.9)$ \\
\hline Lobular & 71 & 7 & 7 & $1.00(0.40,2.06)$ & $90.1(0.035)$ & 91.0 & $0.9(-6.0,7.8)$ \\
\hline
\end{tabular}


Table 4 Observed and predicted 5-year overall survival from therapy calculator, stratified by patients' characteristics (Continued)

\begin{tabular}{|c|c|c|c|c|c|c|c|}
\hline Mucinous & 58 & 1 & 4 & $0.25(0.01,1.39)$ & $98.3(0.017)$ & 96.0 & $-2.3(-5.6,1.0)$ \\
\hline Others & 63 & 8 & 7 & $1.14(0.49,2.25)$ & $88.9(0.040)$ & 89.7 & $0.8(-7.0,8.6)$ \\
\hline \multicolumn{8}{|l|}{ Grade } \\
\hline 1 & 161 & 8 & 11 & $0.73(0.31,1.43)$ & $95.0(0.017)$ & 95.6 & $0.6(-2.7,3.9)$ \\
\hline 2 & 661 & 111 & 71 & $1.56(1.29,1.88)$ & $83.2(0.015)$ & 90.5 & $7.3(4.4,10.2)$ \\
\hline 3 & 433 & 119 & 59 & $2.02(1.67,2.41)$ & $72.5(0.021)$ & 87.7 & $15.2(11.1,19.3)$ \\
\hline Unknown & 283 & 48 & 32 & $1.50(1.11,1.99)$ & $83.0(0.022)$ & 89.8 & $6.8(2.5,11.1)$ \\
\hline \multicolumn{8}{|l|}{ Chemo-therapy } \\
\hline No chemo-therapy & 440 & 58 & 53 & $1.09(0.83,1.41)$ & $86.8(0.016)$ & 90.4 & $3.6(0.5,6.7)$ \\
\hline $1^{\text {st }}$ Gen & 162 & 49 & 21 & $2.33(1.73,3.08)$ & $69.8(0.036)$ & 88.1 & $18.3(11.2,25.4)$ \\
\hline $2^{\text {nd }}$ Gen & 915 & 174 & 97 & $1.79(1.54,2.08)$ & $81.0(0.013)$ & 90.0 & $9.0(6.5,11.5)$ \\
\hline $3^{\text {rd }}$ Gen & 21 & 5 & 2 & $2.50(0.81,5.83)$ & $76.2(0.093)$ & 90.8 & $14.6(-3.6,32.8)$ \\
\hline \multicolumn{8}{|l|}{ Hormone-therapy } \\
\hline No & 398 & 108 & 51 & $2.12(1.74,2.56)$ & $72.9(0.022)$ & 87.7 & $14.8(10.5,19.1)$ \\
\hline Yes & 1140 & 178 & 122 & $1.46(1.25,1.69)$ & $84.4(0.011)$ & 90.8 & $6.4(4.2,8.6)$ \\
\hline
\end{tabular}

Numbers marked in bold indicate statistically significant difference at the $95 \%$ confidence level

number of deaths (752 vs $667, \mathrm{MR}=1.13,95 \%$ CI $1.05-$ 1.21). The number of observed and predicted number of deaths within 10 years after diagnosis was not significant ( 488 vs $454, \mathrm{MR}=1.07,95 \%$ CI $0.98-1.17$ ). The absolute differences of 5-year and 10-year predicted and observed survival probabilities were $3.9 \%$ and $4.9 \%$. Overestimation was more pronounced in Malaysian patients than in Singaporean patients (5.8\% vs $2.5 \%$ for 5 -year survival, and $8.0 \%$ vs $0.0 \%$ for 10 -year survival). We also observed notable differences for cases diagnosed in earlier period and of younger age (Tables 2 and 3 ). In addition, CancerMath significantly overpredicted survival for patients with unfavorable prognostic characteristics such as large tumor size, more positive nodes and ER negative tumor. For those with relatively better predicted survival, CancerMath predictions were similar to observed outcome (Fig. 2a, b and c). The difference between 5 -year predicted and observed survival was $15 \%, 3 \%$ and $1 \%$ for the first, fifth, and tenth deciles respectively. The Kaplan-Meier curves of overall survival by quintiles of predicted 5-year survival were illustrated in Fig. 3. The difference in survival experience between the five groups was statistically significant ( $p$-value $<0.001$ by the log-rank test). The AUC for 5-year and 10-year overall survival were 0.77 (95\% CI,0.75-0.79) and 0.74 (95\% CI,0.71-0.76), respectively whereas the c-index was 0.74 ( $95 \% \mathrm{CI}, 0.72$ $0.75)$. Both measures demonstrated fair discrimination.

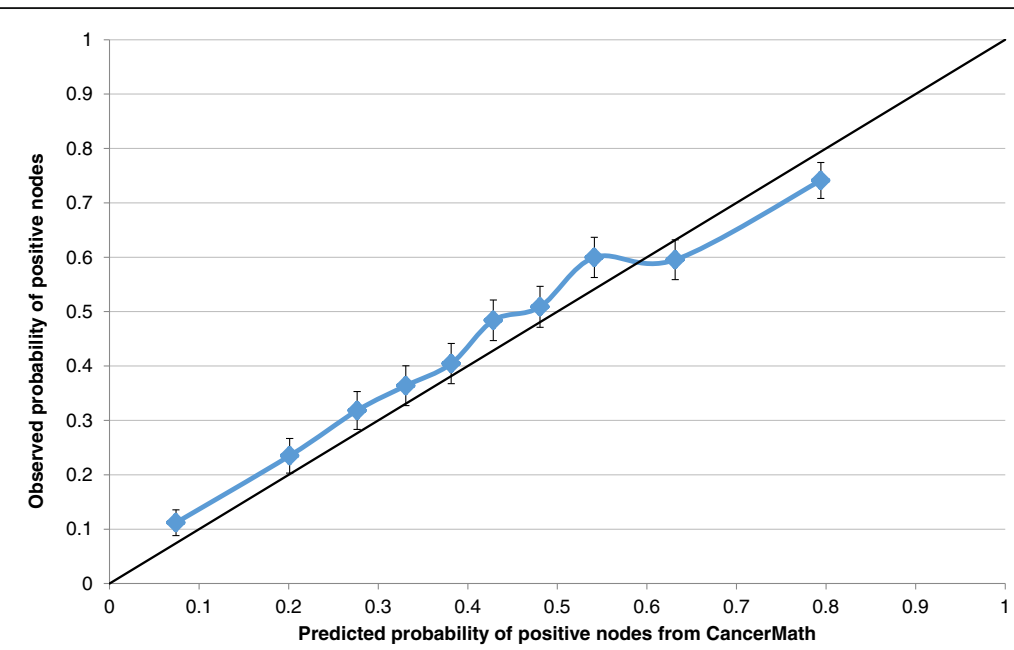

Fig. 1 Calibration plot of observed probability of positive nodes with $95 \%$ confidence interval against predicted probability of positive nodes (mean) by deciles of the predicted value 


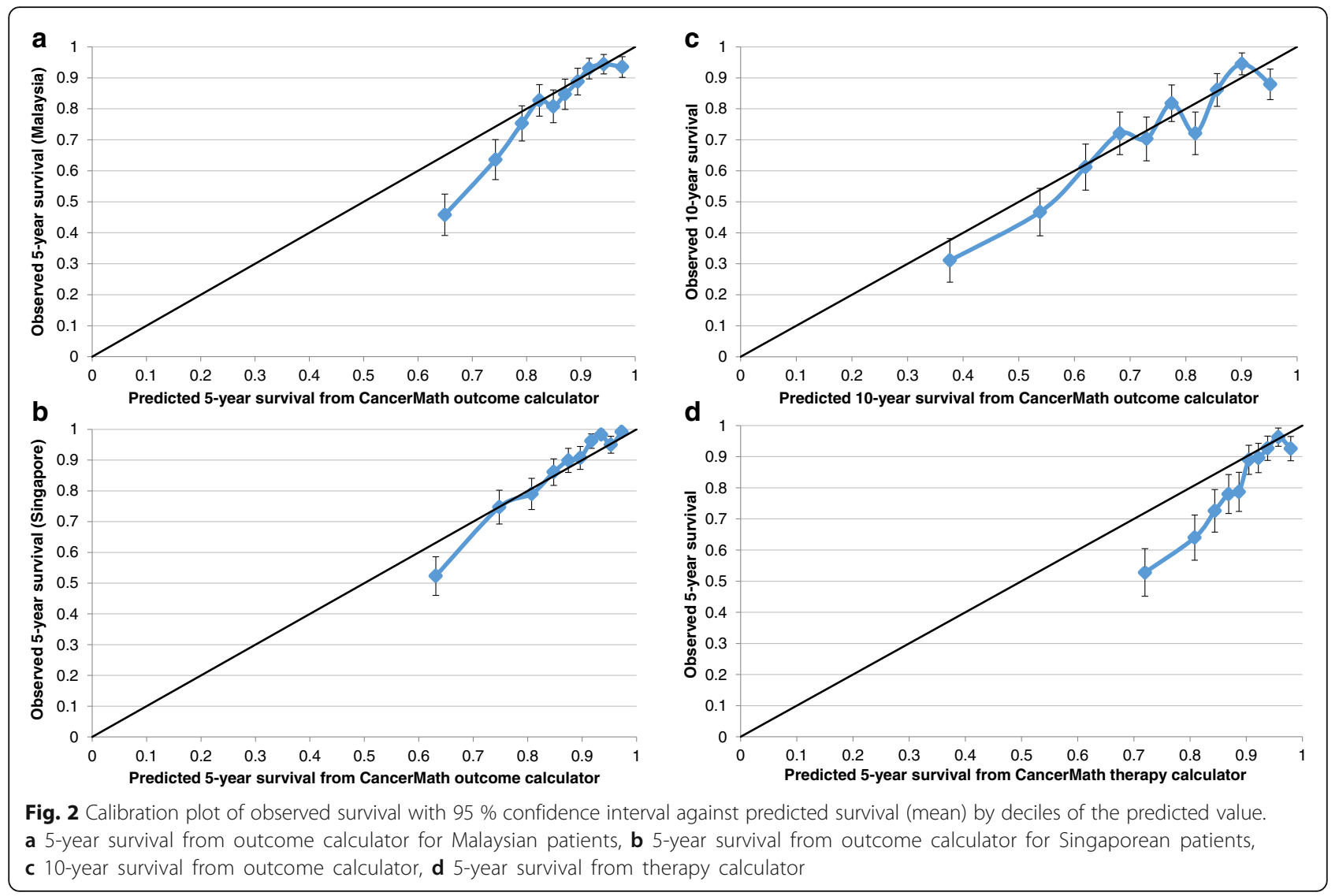

\section{Therapy calculator}

For therapy calculator which was only validated in Malaysian patients, predicted survival was significantly higher than the observed survival for almost all subgroups, except for those diagnosed recently and with more favorable tumor characteristics (Table 4, Fig. 2d). The calculator showed fair discrimination at 5-year overall survival (AUC $=0.73,95 \%$ CI 0.70-0.77).

\section{Conditional survival calculator}

For patients who have survived 2 years since diagnosis, the predicted 5-year survival was $91.0 \%$ versus the observed survival of $88.3 \%$. The AUC was 0.75 (95\% CI, $0.73-0.77$ ). For patients who have survived 5 years and 7 years, the predicted probability of surviving up to 10 years was $86.6 \%$ and $91.7 \%$. Whereas the observed survival was $85.3 \%$ and $91.0 \%$ correspondingly. The AUC was 0.66 (95\% CI, 0.62-0.70) and 0.63 (95\% CI, $0.57-0.68$ ) for 10 -year survival.

\section{Discussion}

Many prognostic tools have been developed over the past two decades to aid clinical decision making for breast cancer patients. This study validated four different prognostic calculators provided by CancerMath in the Singapore-Malaysia Hospital-Based Breast Cancer
Registry. The discrimination was fair for nodal status calculator. CancerMath outcome, therapy and conditional survival calculator also moderately discriminated between survivors and non-survivors at 5 years and 10 years after diagnosis. It however consistently overestimated survival for this cohort of Southeast Asian patients, especially for those with poor prognostic profile.

CancerMath was previously built and validated using SEER data and patients diagnosed at Massachusetts General and Brigham and Women's Hospitals [19]. In the SEER database, $82.7 \%$ of the invasive breast cancer cases diagnosed between 2003 and 2007 were white and only $6.9 \%$ were Asian/ /Pacific Islander [28]. It was shown that the differences between observed and predicted survival was within $2 \%$ for $97 \%$ of the patients in the validation set [19]. Our study is the first one to independently validate CancerMath outside United States and is also the largest validation study of a westernderived breast cancer prognostic model in Asia. We demonstrated that CancerMath overpredicted survival by more than $3 \%$ for almost all clinical and pathological subgroups. The findings were similar to previous validation studies of Adjuvant! Online conducted in Asia. In the Malaysian, Korean, and Taiwanese studies, the predicted and observed 10-year overall survival differed by $6.7 \%, 11.1 \%$, and $3.9 \%$ correspondingly [16-18]. The 


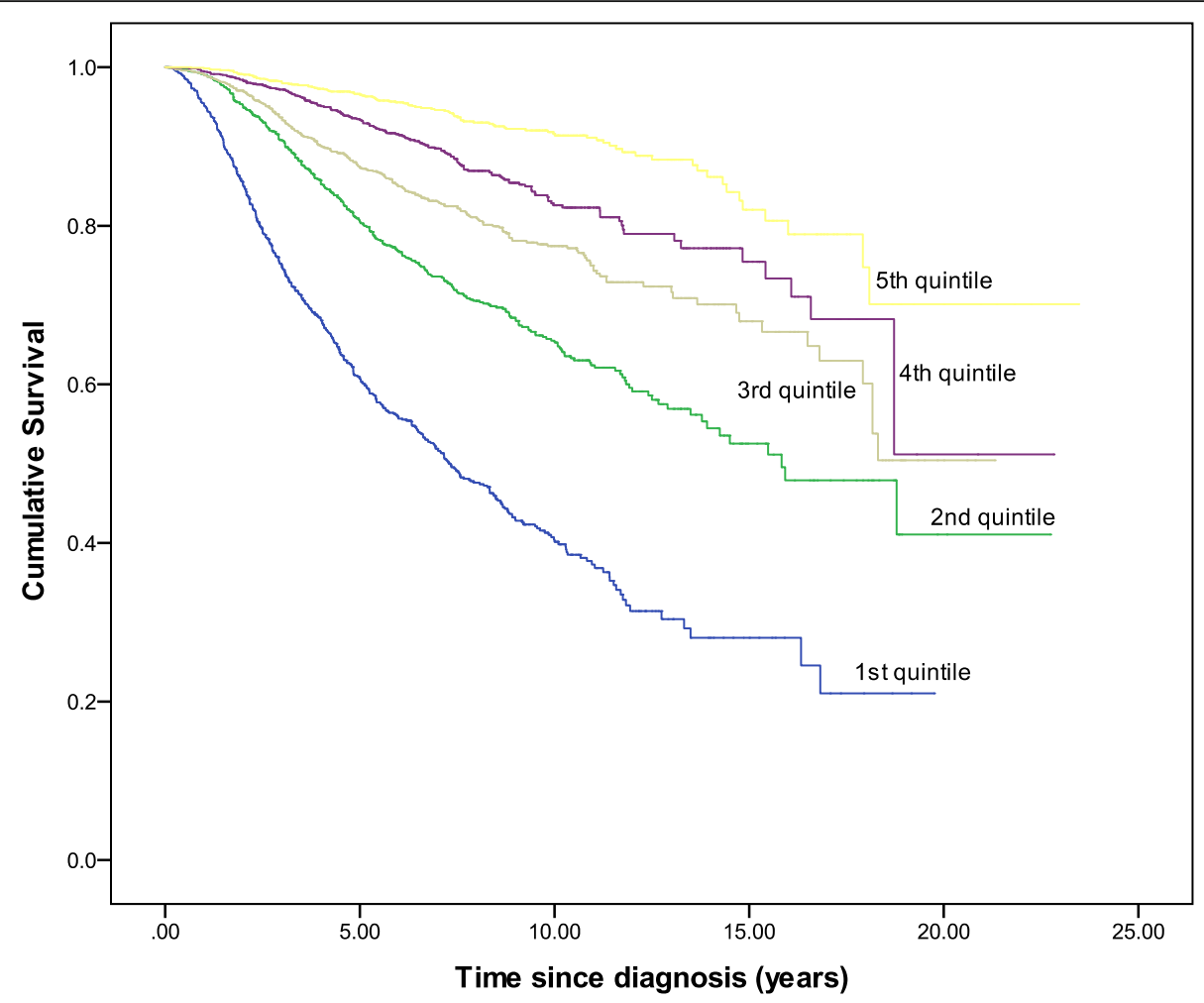

Fig. 3 Kaplan-Meier curves of overall survival by quintiles of 5-year predicted survival from outcome calculator

AUC of Adjuvant! Online was 0.73 (95\% CI, 0.69-0.77) in the Malaysian study and hence very close to the AUC of CancerMath reported in the present study [16]. Furthermore the prediction was too optimistic for young patients in almost all validation studies of Adjuvant! Online [12, 15-17]. Although adjustment of 1.5-fold increase in risk was added to Adjuvant! Online version 7.0 for patients younger than 36 years and with ER positive breast cancer, overprediction was still found in recent validation studies [12, 16, 17]. Our findings from current validation of CancerMath also suggested that correction for young age at diagnosis is needed.

The selection of patients for validation can partially explain the discrepancy in observed and predicted survival. CancerMath has only been validated among patients with tumor size no more than $50 \mathrm{~mm}$ and positive nodes no more than seven [29]. In our validation dataset, $10 \%$ of patients had tumor size larger than $50 \mathrm{~mm}$ and $8 \%$ had more than ten positive nodes. However even for patients with tumor size in between $20 \mathrm{~mm}$ and $50 \mathrm{~mm}$ and one to three positive nodes, the difference between the predicted and observed survival was more than $3 \%$. In general, Asian patients are more likely to present with unfavorable prognostic features such as young age, negative hormone receptor status, HER2 overexpression, and more advanced stage compared to their western counterparts [30-32]. In our current analysis, reduced agreement was observed for patients with poorer predicted outcome, especially for Malaysian patients, as illustrated by the calibration plot. In addition, the slope of the calibration plot for Malaysian patients were greater than 1 for the first three deciles which suggested that the spread of the predicted survival was less than observed survival. CancerMath's poorer performance in Malaysia might be explained by higher proportion of patients in advanced stages and more heterogeneous prognosis in Malaysia. Such limitation of CancerMath may restrict its use to patients with better prognostic profile only. Furthermore CancerMath therapy calculator applies the same amount of risk reduction from adjuvant therapy as Adjuvant! Online, which was estimated from meta-analysis of clinical trials mainly conducted in western population $[9,19]$. However nonadherence to treatment is more common among Asian women [33-35]. Studies also reported different drug metabolism and toxicity induced by chemotherapy between Asian and Caucasian patients [36]. These evidences may imply CancerMath overestimate the effect of treatment in Asian patients.

Another possible explanation of suboptimal performance of CancerMath and also the limitation of our study is missing data on ER (6\%), PR (15\%), HER2 status (47 \%), and tumor grade (11\%). For patients with complete information on required predictors $(N=1872)$, 
the predicted and observed 5-year survival was $86.0 \%$ and $82.5 \%$. The difference were similar to what we observed in the entire dataset. Therefore the impact of missing data is relatively small on performance of CancerMath.

Several gene expression profiling assays, such as MammaPrint [37] and Oncotype Dx [38] are currently available for breast cancer prognostication and treatment decision. However these tools do not incorporate clinicopathologic factors which are readily available or relatively cheap to obtain. Due to the high cost of these tests and larger proportion of patients with high predicted risk in Asia $[39,40]$, the clinical utility is uncertain in this region. Therefore traditional prognostic model using clinicopathologic factors seems more reasonable in our local setting.

\section{Conclusions}

In conclusion, CancerMath demonstrated modest discrimination and calibration among Southeast Asian patients. Our results suggest that CancerMath is more suitable for patients diagnosed with favorable disease.

\section{Abbreviations}

AC: Doxorubicin and cyclophosphamide; Al: Aromatase inhibitors; AUC: Area under the curve; $\mathrm{Cl}$ : Confidence interval; C-index: Concordance index; CMF: Cyclophosphamide, methotrexate and fluorouracil; ER: Estrogen receptor; FEC: Fluorouracil, epirubicin and cyclophosphamide; FISH: Fluorescence in situ hybridization; HER2: Human epidermal growth factor receptor 2; IHC: Immunohistochemistry; MR: Mortality ratio; NUH: National University Hospital; PR: Progesterone receptor; SEER: Surveillance, epidemiology and end-result; TAC: Docetaxel, doxorubicin and cyclophosphamide; TTSH: Tan Tock Seng Hospital; UMMC: University Malaya Medical Centre.

\section{Acknowledgement}

Not applicable.

\section{Funding}

This study was supported by Clinician Scientist Award (NMRC/CSA/0048/ 2013) from National Medical Research Council, Singapore and a High Impact Research Grant (UM.C/HIR/MOHE/06) from the Ministry of Higher Education, Malaysia.

\section{Availability of data and material}

The datasets generated during and/or analysed during the current study are not publicly available due to potential breach of confidentiality but are available from the corresponding author on reasonable request.

\section{Author's contributions}

$\mathrm{HM}, \mathrm{MH}, \mathrm{HMV}$ and NB were involved in study design, analysis and interpretation of data, and manuscript writing. NAT, HW, SS, CY, ET, PC and SL collected and interpreted data, critically reviewed the manuscript and provided suggestion for revision. All authors read and approved the final report before submission.

\section{Competing interests}

The authors declare that they have no competing interests.

\section{Consent for publication}

Not applicable.

\section{Ethics approval and consent to participate}

Ethics approval was obtained from Domain Specific Review Board under National Healthcare Group in Singapore and Medical Ethics Committee under University Malaya Medical Centre.

\section{Author details}

${ }^{1}$ Saw Swee Hock School of Public Health, National University of Singapore and National University Health System, Tahir Foundation Building, 12 Science Drive 2, Singapore 117549, Singapore. ${ }^{2}$ Department of Surgery, National University Hospital, 1E Kent Ridge Road, Singapore 119228, Singapore. ${ }^{3}$ Department of Medical Epidemiology and Biostatistics, Karolinska Institutet, PO Box 281, SE-171 77 Stockholm, Sweden. ${ }^{4}$ Imaging Division, University Medical Center Utrecht, PO Box 85500 3508, GA, Utrecht, The Netherlands. ${ }^{5}$ Department of Surgery, Faculty of Medicine, University of Malaya, 50603 Kuala Lumpur, Malaysia. ${ }^{6}$ Clinical Epidemiology Unit, National Clinical Research Centre, Jalan Pahang, 50586 Kuala Lumpur, Malaysia. ${ }^{7}$ Department of Surgery, Tan Tock Seng Hospital, 11 Jalan Tan Tock Seng, Singapore 308433, Singapore. ${ }^{8}$ Department of Hematology Oncology, National University Cancer Institute, National University Health System, 1 E Kent Ridge Road, Singapore 119228, Singapore. ${ }^{9}$ Department of Social and Preventive Medicine, Faculty of Medicine, University of Malaya, 50603 Kuala Lumpur, Malaysia. ${ }^{10}$ Julius Center for Health Sciences and Primary Care, University Medical Center, PO Box 85500 3508, AB, Utrecht, The Netherlands.

Received: 16 December 2015 Accepted: 5 October 2016 Published online: 21 October 2016

\section{References}

1. Early Breast Cancer Trialists' Collaborative G. Effects of chemotherapy and hormonal therapy for early breast cancer on recurrence and 15-year survival: an overview of the randomised trials. Lancet. 2005:365(9472):1687-717.

2. Clarke M. Meta-analyses of adjuvant therapies for women with early breast cancer: the Early Breast Cancer Trialists' Collaborative Group overview. Ann Oncol. 2006;17 Suppl 10:x59-62.

3. Early Breast Cancer Trialists' Collaborative G, Clarke M, Coates AS, Darby SC, Davies C, Gelber RD, Godwin J, Goldhirsch A, Gray R, Peto R, et al. Adjuvant chemotherapy in oestrogen-receptor-poor breast cancer: patient-level metaanalysis of randomised trials. Lancet. 2008;371(9606):29-40.

4. Bertos NR, Park M. Breast cancer - one term, many entities? J Clin Invest. 2011;121(10):3789-96.

5. Higgins MJ, Baselga J. Targeted therapies for breast cancer. J Clin Invest. 2011:121(10):3797-803.

6. Engelhardt EG, Garvelink MM, de Haes JH, van der Hoeven JJ, Smets EM, Pieterse AH, Stiggelbout AM. Predicting and communicating the risk of recurrence and death in women with early-stage breast cancer: a systematic review of risk prediction models. J Clin Oncol. 2014;32(3):238-50.

7. Haybittle JL, Blamey RW, Elston CW, Johnson J, Doyle PJ, Campbell FC, Nicholson Rl, Griffiths K. A prognostic index in primary breast cancer. Br J Cancer. 1982:45(3):361-6.

8. Todd JH, Dowle C, Williams MR, Elston CW, Ellis IO, Hinton CP, Blamey RW, Haybittle JL. Confirmation of a prognostic index in primary breast cancer. $\mathrm{Br}$ J Cancer. 1987;56(4):489-92.

9. Ravdin PM, Siminoff LA, Davis GJ, Mercer MB, Hewlett J, Gerson N, Parker $\mathrm{HL}$. Computer program to assist in making decisions about adjuvant therapy for women with early breast cancer. J Clin Oncol. 2001;19(4):980-91.

10. Harnett A, Smallwood J, Titshall V, Champion A, Guideline Development G. Diagnosis and treatment of early breast cancer, including locally advanced disease-summary of NICE guidance. BMJ. 2009;338:b438.

11. Agarwal V, O'Neill P. Adjuvant! Online as a decision-making tool in early breast cancer-a UK national survey. Clin Oncol. 2011;23(2):159-60.

12. Mook S, Schmidt MK, Rutgers EJ, van de Velde AO, Visser O, Rutgers SM Armstrong N, van't Veer $L$, Ravdin PM. Calibration and discriminatory accuracy of prognosis calculation for breast cancer with the online Adjuvant! program: a hospital-based retrospective cohort study. Lancet Oncol. 2009;10(11):1070-6.

13. Epstein RJ, Leung TW, Mak J, Cheung PS. Utility of a web-based breast cancer predictive algorithm for adjuvant chemotherapeutic decision making in a multidisciplinary oncology center. Cancer Investig. 2006;24(4):367-73.

14. Campbell HE, Taylor MA, Harris AL, Gray AM. An investigation into the performance of the Adjuvant! Online prognostic programme in early breast cancer for a cohort of patients in the United Kingdom. Br J Cancer. 2009;101(7):1074-84.

15. Olivotto IA, Bajdik CD, Ravdin PM, Speers CH, Coldman AJ, Norris BD, Davis GJ, Chia SK, Gelmon KA. Population-based validation of the prognostic model ADJUVANT! for early breast cancer. J Clin Oncol. 2005;23(12):2716-25. 
16. Bhoo-Pathy N, Yip CH, Hartman M, Saxena N, Taib NA, Ho GF, Looi LM, Bulgiba AM, van der Graaf $Y$, Verkooijen HM. Adjuvant! Online is overoptimistic in predicting survival of Asian breast cancer patients. Eur J Cancer. 2012:48(7):982-9.

17. Jung M, Choi EH, Nam CM, Rha SY, Jeung HC, Lee SH, Yang WI, Roh JK, Chung HC. Application of the adjuvant! Online model to Korean breast cancer patients: an assessment of prognostic accuracy and development of an alternative prognostic tool. Ann Surg Oncol. 2013;20(8):2615-24.

18. Yao-Lung K, Dar-Ren C, Tsai-Wang C. Accuracy validation of adjuvant! online in Taiwanese breast cancer patients-a 10-year analysis. MC Med Inform Decis Mak. 2012;12:108.

19. Michaelson JS, Chen LL, Bush D, Fong A, Smith B, Younger J. Improved web-based calculators for predicting breast carcinoma outcomes. Breast Cancer Res Treat. 2011;128(3):827-35.

20. Chen LL, Nolan ME, Silverstein MJ, Mihm Jr MC, Sober AJ, Tanabe KK, Smith BL, Younger J, Michaelson JS. The impact of primary tumor size, lymph node status, and other prognostic factors on the risk of cancer death. Cancer. 2009;115(21):5071-83.

21. Agarwal G, Pradeep PV, Aggarwal V, Yip CH, Cheung PS. Spectrum of breast cancer in Asian women. World J Surg. 2007;31(5):1031-40.

22. Porter P. "Westernizing" women's risks? Breast cancer in lower-income countries. N Engl J Med. 2008;358(3):213-6.

23. Kamangar F, Dores GM, Anderson WF. Patterns of cancer incidence, mortality, and prevalence across five continents: defining priorities to reduce cancer disparities in different geographic regions of the world. J Clin Oncol. 2006;24(14):2137-50.

24. Pathy NB, Yip CH, Taib NA, Hartman M, Saxena N, lau P, Bulgiba AM, Lee SC, Lim SE, Wong JE, et al. Breast cancer in a multi-ethnic Asian setting: results from the Singapore-Malaysia hospital-based breast cancer registry. Breast. 2011;20 Suppl 2:S75-80.

25. Liddell FD. Simple exact analysis of the standardised mortality ratio. J Epidemiol Community Health. 1984;38(1):85-8.

26. Wishart GC, Bajdik CD, Azzato EM, Dicks E, Greenberg DC, Rashbass J, Caldas C, Pharoah PD. A population-based validation of the prognostic model PREDICT for early breast cancer. Eur J Surg Oncol. 2011;37(5):411-7.

27. Harrell Jr FE, Califf RM, Pryor DB, Lee KL, Rosati RA. Evaluating the yield of medical tests. JAMA. 1982;247(18):2543-6.

28. Altekruse SF, Kosary CL, Krapcho M, Neyman N, Aminou R, Waldron W, Ruhl J, Howlader N, Tatalovich Z, Cho H, Mariotto A, Eisner MP, Lewis DR, Cronin K, Chen HS, Feuer EJ, Stinchcomb DG, Edwards BK (eds): SEER Cancer Statistics Review, 1975-2007, National Cancer Institute. Bethesda, MD, http://www.seer.cancer.gov/csr/1975_2007/, based on November 2009 SEER data submission, posted to the SEER web site, 2010. Accessed 2 Sept 2016.

29. Chen LL, Bush D, Fong A, Tanabe KK, Younger J, Michaelson JS: Technical Repor \#10 -Technical report for the paper: "CancerMath.net: Wed-based Calculators for Breast Carcinoma" (July 28, 2009, updated November 28, 2009 and March 22, 2010) at http://cancer.lifemath.net/about/techreports/ index.php In.; 2010.

30. Tan BK, Lim GH, Czene K, Hall P, Chia KS. Do Asian breast cancer patients have poorer survival than their western counterparts? A comparison between Singapore and Stockholm. Breast Cancer Res. 2009;11(1):R4.

31. Lim SE, Back M, Quek E, lau P, Putti T, Wong JE. Clinical observations from a breast cancer registry in Asian women. World J Surg. 2007;31(7):1387-92.

32. Telli ML, Chang ET, Kurian AW, Keegan TH, McClure LA, Lichtensztajn D, Ford JM, Gomez SL. Asian ethnicity and breast cancer subtypes: a study from the California Cancer Registry. Breast Cancer Res Treat. 2011;127(2):471-8.

33. Iskandarsyah A, de Klerk C, Suardi DR, Soemitro MP, Sadarjoen SS, Passchier J. Psychosocial and cultural reasons for delay in seeking help and nonadherence to treatment in Indonesian women with breast cancer: a qualitative study. Health Psychol. 2014;33(3):214-21.

34. Leong BD, Chuah JA, Kumar VM, Rohamini S, Siti ZS, Yip CH. Trends of breast cancer treatment in Sabah, Malaysia: a problem with lack of awareness. Singap Med J. 2009;50(8):772-6.

35. Chong HY, Taib NA, Rampal S, Saad M, Bustam AZ, Yip CH. Treatment options for locally advanced breast cancer-experience in an Asian tertiary hospital. Asian Pac J Cancer Prev. 2010;11(4):913-7.

36. Phan VH, Moore MM, McLachlan AJ, Piquette-Miller M, Xu H, Clarke SJ. Ethnic differences in drug metabolism and toxicity from chemotherapy. Expert Opin Drug Metab Toxicol. 2009:5(3):243-57.
37. Veer $L J \vee$ 't, Dai $H$, van de Vijver MJ, He YD, Hart AA, Mao M, Peterse HL, Van der Kooy K, Marton MJ, Witteveen AT, et al. Gene expression profiling predicts clinical outcome of breast cancer. Nature. 2002;415(6871):530-6.

38. Paik S, Shak S, Tang G, Kim C, Baker J, Cronin M, Baehner FL, Walker MG, Watson D, Park T, et al. A multigene assay to predict recurrence of tamoxifentreated, node-negative breast cancer. N Engl J Med. 2004;351(27):2817-26.

39. Na KY, Kim KS, Lee JE, Kim HJ, Yang JH, Ahn SH, Moon BI, Kim RM, Ko SM, Jung YS. The 70-gene prognostic signature for korean breast cancer patients. J Breast Cancer. 2011;14(1):33-8.

40. Ishitobi M, Goranova TE, Komoike Y, Motomura K, Koyama H, Glas AM, van Lienen E, Inaji H, Van't Veer $\sqcup$, Kato K. Clinical utility of the 70-gene MammaPrint profile in a Japanese population. Jpn J Clin Oncol. 2010;40(6):508-12.

\section{Submit your next manuscript to BioMed Central and we will help you at every step:}

- We accept pre-submission inquiries

- Our selector tool helps you to find the most relevant journal

- We provide round the clock customer support

- Convenient online submission

- Thorough peer review

- Inclusion in PubMed and all major indexing services

- Maximum visibility for your research

Submit your manuscript at www.biomedcentral.com/submit
C) Biomed Central 